\title{
Efficacy of a novel topical combination of esafoxolaner, eprinomectin and praziquantel against Rhipicephalus sanguineus in cats
}

\author{
Eric Tielemans ${ }^{1, *}$, Anthony Pfefferkorn ${ }^{1}$, and Alta Viljoen ${ }^{2}$ \\ ${ }^{1}$ Boehringer-Ingelheim Animal Health, 29 avenue Tony Garnier, 69007 Lyon, France \\ 2 Clinvet International (Pty) Ltd., P.O. Box 11186, Universitas, Bloemfontein 9321, Republic of South Africa
}

Received 13 December 2019, Accepted 8 March 2021, Published online 2 April 2021

\begin{abstract}
Esafoxolaner is a purified enantiomer of afoxolaner with insecticidal and acaricidal properties. It is combined with eprinomectin and praziquantel in a novel topical endectoparasiticide formulation for cats. The efficacy of this novel formulation was assessed in an experimental study against induced infestation of Rhipicephalus sanguineus ticks. Twenty cats were randomly allocated to either a placebo control group or a treated group in a 1:1 ratio. Infested cats were treated topically once at the minimum recommended dose. The study was designed to assess curative efficacy $48 \mathrm{~h}$ after treatment and to test preventive efficacy $48 \mathrm{~h}$ after weekly infestations for 2 months. At each weekly infestation, all cats were infested with 25 male and 25 unfed female $R$. sanguineus ticks. At each tick count, at least 6 in 10 control cats had a retention of $13(26 \%)$ or more live ticks, demonstrating adequate infestation throughout the study. Curative efficacy on existing tick infestation was $90 \%$; preventive efficacy over the following 6 weeks was at least $96 \%$.
\end{abstract}

Key words: Cat, Esafoxolaner, Tick, Rhipicephalus sanguineus, Efficacy.

Résumé - Efficacité d'une nouvelle association topique d'esafoxolaner, d'éprinomectine et de praziquantel contre Rhipicephalus sanguineus chez le chat. L'esafoxolaner est un énantiomère purifié d'afoxolaner, aux propriétés insecticides et acaricides. Il est combiné à éprinomectine et praziquantel dans une nouvelle formulation topique endectoparasiticide pour chats. L'efficacité de cette nouvelle formulation a été testée lors d'une étude contre des infestations expérimentales avec des tiques Rhipicephalus sanguineus. Vingt chats ont été répartis au hasard soit dans un groupe témoin placebo soit dans un groupe traité (rapport 1:1). Les chats infestés ont été traités par voie topique une fois à la dose minimale recommandée. L'étude a été conçue pour une évaluation de l'efficacité curative 48 heures après traitement et pour des évaluations d'efficacité préventive 48 heures après chaque infestation hebdomadaire pendant 2 mois. À chaque infestation hebdomadaire, tous les chats étaient infestés par 25 mâles et 25 femelles de $R$. sanguineus, non nourris. À chaque comptage, au moins 6 chats sur 10 du groupe placebo contrôle étaient infestés avec au moins 13 (26\%) tiques vivantes, ce qui a validé le modèle d'infestation. L'efficacité curative sur tiques présentes avant traitement a été de $90 \%$, l'efficacité préventive durant les six semaines suivantes a été d'au moins $96 \%$.

\section{Introduction}

Rhipicephalus sanguineus, the brown dog tick is the most widespread tick species in the world. It affects dogs living in urban and rural areas, and throughout the year [5, 10]. The main host of $R$. sanguineus is the dog, on which it can complete its full development cycle, but this species also infests other mammals, including cats, rodents, livestock and humans at immature or adult stages $[5,8,9]$. Rhipicephalus sanguineus is an important vector of a diverse range of pathogens, such as Babesia, Cercopithifilaria, Hepatozoon, Ehrlichia, and Rickettsia [1-5, $9,14,18]$. Rhipicephalus sanguineus is highly adapted to warm conditions in tropical and subtropical climates, either dry or wet, but can also thrive in temperate climates where global warming contributes to its increased presence and relevance from a public health perspective [5]. Rhipicephalus sanguineus

*Corresponding author: eric.tielemans@boehringer-ingelheim.com

Special Issue - NexGard ${ }^{\circledR}$ Combo (esafoxolaner, eprinomectin, praziquantel): A new endectocide spot-on formulation for cats. Invited Editor: Frédéric Beugnet

This is an Open Access article distributed under the terms of the Creative Commons Attribution License (https://creativecommons.org/licenses/by/4.0), which permits unrestricted use, distribution, and reproduction in any medium, provided the original work is properly cited. 
Table 1. Rhipicephalus sanguineus, mean live tick counts per group and efficacy results.

\begin{tabular}{|c|c|c|c|c|c|c|c|c|c|c|c|}
\hline & & $n^{5}$ & Day 2 & Day 9 & Day 16 & Day 23 & Day 32 & Day 39 & Day 46 & Day 55 & Day 60 \\
\hline Control group ${ }^{1}$ & $\mathrm{AM}^{3}$ & 10 & 15.7 & 19.5 & 21.9 & 18.5 & 19.2 & 15.7 & 17.9 & 16.9 & 14.2 \\
\hline \multirow[t]{3}{*}{ Treated group ${ }^{2}$} & $\mathrm{AM}^{3}$ & 10 & 1.6 & 0.0 & 0.0 & 0.0 & 0.2 & 0.5 & 0.7 & 2.0 & 5.3 \\
\hline & & $\%$ Efficacy $^{4}$ & 89.8 & 100.0 & 100.0 & 100.0 & 99.0 & 96.8 & 96.1 & 88.2 & 62.7 \\
\hline & & $p$-value & $<0.0001$ & $<0.0001$ & $<0.0001$ & $<0.0001$ & $<0.0001$ & $<0.0001$ & $<0.0001$ & $<0.0001$ & 0.0191 \\
\hline
\end{tabular}

${ }^{1}$ The Control (CP) group was treated topically once on Day 0 with $0.12 \mathrm{~mL} / \mathrm{kg}$ Technical oil.

${ }^{2}$ The Treated (IVP) group was treated topically once on Day 0 with $0.12 \mathrm{~mL} / \mathrm{kg}$ of the novel formulation of (S)afoxolaner, eprinomectin and praziquantel.

${ }^{3}$ Percent efficacy $=[(C-T) / C] \times 100$, where $\mathrm{T}$ and $\mathrm{C}$ are arithmetic means (AM) of the IVP group and the CP group, respectively.

${ }_{5}^{4} p$-value $=$ two-sided $p$-value from the MIXED model of the IVP group and the CP group.

5 number of cats per group: one cat was removed on Day 50 because deemed unsuitable to remain in the study for excessive tick bite wounds, consequently $n=9$ in the control group on Days 55 and 60.

is not a very common tick species infesting cats, like Ixodes spp. or Amblyomma americanum; however, it is occasionally described on cats, especially in the presence of dogs $[5,16$, 17]. The behavior of free-roaming cats including predation of infested rodents, access to hidden places where immature $R$. sanguineus stages moult, and movements may expose cats to immature $R$. sanguineus infestation [11]. Furthermore, the grooming habits of cats allow them to remove larger adult ticks more easily than small larvae or nymphs, which might be another explanation as to why a larger proportion of immature stages than adults was found on cats [11].

A novel topical combination of esafoxolaner, eprinomectin and praziquantel was developed to offer a wide parasiticide spectrum and integrated control of cat parasites. Afoxolaner has already been proven effective against $R$. sanguineus in dogs [12]. Afoxolaner is a racemic mixture, and esafoxolaner is the active purified enantiomer. This article describes a study performed to evaluate the efficacy of this novel formulation for the treatment and control of $R$. sanguineus infestations in cats.

\section{Materials and methods \\ Study design}

The study was designed in accordance with the "World Association for the Advancement of Veterinary Parasitology (W.A.A.V.P.) guidelines for evaluating the efficacy of parasiticides for the treatment, prevention and control of flea and tick infestation on dogs and cats" [13]. It was conducted in accordance with Good Clinical Practices as described in International Cooperation on Harmonization of Technical Requirements for Registration of Veterinary Medicinal Products (VICH) guideline GL9. The study protocol had been reviewed and approved by the Sponsor's and local Institutional Animal Care and Use Committees. Cats were handled with due regard for their wellbeing.

The study was conducted under a negative-controlled and randomized design. Randomization was based on a pre-treatment live $R$. sanguineus count, $48 \mathrm{~h}$ after infestation. The efficacy assessment was based on comparison of live ticks found in the control and treated groups at identical weekly time-points after treatment.
The study was performed in South Africa, during OctoberDecember 2018. All personnel collecting animal health and efficacy data were blinded to treatment. Cats were single housed during the study to avoid inter-animal treatment contamination.

\section{Animals}

Twenty purpose-bred, healthy laboratory shorthair cats, 10 males and 10 females, aged 6 months -5 years old and weighing $1.9-5.3 \mathrm{~kg}$ were included in the study.

\section{Rhipicephalus sanguineus strain}

The $R$. sanguineus originated from the field in 2011 in Georgia, United States. The colony had been maintained at the test facility since 2014 on rabbits not treated with acaricides.

\section{Treatment}

Cats were treated once on Day 0. The treatment was applied topically on the skin, after parting the hair, on one spot in the midline of the neck between the base of the skull and the shoulder blades. Cats assigned to the placebo control group were treated with mineral oil at $0.12 \mathrm{~mL} / \mathrm{kg}$, cats assigned to the treated group were applied the novel formulation at the minimum recommended dose of $0.12 \mathrm{~mL} / \mathrm{kg}$, delivering $1.44 \mathrm{mg} / \mathrm{kg}$ esafoxolaner, $0.48 \mathrm{mg} / \mathrm{kg}$ eprinomectin, and $10.0 \mathrm{mg} / \mathrm{kg}$ praziquantel.

To detect the presence or absence of any treatment-related or unrelated health abnormality, health observations were conducted daily and at hourly intervals for $4 \mathrm{~h}$ after treatment.

\section{Tick infestations}

Each cat was infested in a random order, on Days $-2,7,14$, $21,30,37,44,53$ and 58. Animals were sedated and placed in an infestation chamber equipped with adhesive tapes at the edges to act as a tick barrier. Once sedated, cats were equipped with an Elizabethan collar to limit grooming. Twenty-five female and 25 male $R$. sanguineus were then placed on the 
lateral side of each cat and care was taken to avoid the treatment application site. After a maximum of $4 \mathrm{~h}$, cats were removed from the crates and the remaining free ticks placed back on the cat. The Elizabethan collar was left until the tick count procedure.

\section{Tick counts}

Tick counts were performed in a random order. Attached and unattached live and dead ticks were removed and counted approximately $48 \mathrm{~h}$ after treatment on Day 2, and approximately $48 \mathrm{~h}$ after the subsequent infestations on Days 9, 16, $23,32,39,46,55$ and 60 . Tick counts were performed by parting and feeling through the cat's hair with finger tips. When a tick was detected, the hair was further parted, visual confirmation of the tick's presence was made, the tick was removed, and the live or dead status of the tick confirmed. After an area was cleared by this method, the area was combed using a finetoothed flea comb for a second check for tick presence. Each tick was observed for signs of viability or mortality. Viability was evaluated if necessary by breathing on the motionless tick and observing the presence or absence of reaction to this stimulation. Protective clothing (e.g. gowns/coats, gloves, etc.) and combs were changed between each cat to prevent crosscontamination.

\section{Statistical analysis}

To compute the percent efficacy, the arithmetic mean of the live tick counts was calculated by group at each time-point. Percent efficacy of the treated group with respect to the control group was calculated using the formula $[(C-T) / C] \times 100$, where $C=$ arithmetic mean for the control group, and $T=$ arithmetic mean for the treated group. The log-count of each treated group was compared to the log-count of the control group using an $F$-test adjusted for the allocation blocks used to randomize the animals to the treatment groups at each time-point separately. The mixed procedure in SAS version 9.4 was used for the analysis, with group listed as a fixed effect and the allocation blocks listed as a random effect.

\section{Results}

The individual retention of live ticks on the control cats was 18 ticks on average and ranged from 3 to 38 ticks. At each tick count time-point, at least 6 in 10 control cats had a retention of $13(26 \%)$ or more live ticks, which is considered in the WAAVP guideline [13] as demonstrating adequate infestation throughout the study and vigorous tick population.

The curative efficacy of one application of the novel formulation on existing tick infestation was $90 \%$, the preventive efficacy over the following 6 weeks was at least 96\% (Table 1).

No adverse reactions related to treatment were observed.

\section{Discussion and conclusion}

The results of this study illustrate the high level of efficacy of the novel topical formulation of esafoxolaner, eprinomectin and praziquantel against $R$. sanguineus infestation in cats for curative efficacy of existing infestation within $48 \mathrm{~h}$ of the treatment application, and for preventive efficacy within $48 \mathrm{~h}$ of new infestations for 6 weeks.

Even though $R$. sanguineus is not considered a major tick species of cats, it should not be underestimated, in view of its potential contribution to dog infestation by free roaming and spreading of immature and adult stages, and severe vectorborne pathogen transmission to dogs and also humans.

This novel association of esafoxolaner, eprinomectin and praziquantel offers a broad spectrum of efficacy against the main parasites of cats including ecto- and endoparasites. The control of multiple and various concurrent parasitic infestations by a range of cat parasites is important for cats but also public health $[4,6,7,15]$.

Alongside a high level of efficacy and safety, owner and cat compliance is an important feature of success for this type of therapeutic approach, and the easy conditions of use and of treatment application of this product are necessary to guarantee a high level of compliance.

Acknowledgements. The authors gratefully acknowledge the staff at Clinvet International for conducting the study to a high professional standard.

\section{Conflict of interest}

The work reported herein was funded by BoehringerIngelheim. Eric Tielemans and Anthony Pfefferkorn are current employees of Boehringer-Ingelheim, Alta Viljoen is a current employee of Clinvet International. Other than that, the authors declare no conflict of interest. This document is provided for scientific purposes only. Any reference to a brand or trademark herein is for information purposes only and is not intended for any commercial purposes or to dilute the rights of the respective owners of the brand(s) or trademark(s).

\section{References}

1. Beugnet F. 2013. Guide to vector borne diseases of pets. France: Ed Ferreol. 425 p.

2. Beugnet F, Halos L. 2015. Parasitoses \& vector borne diseases of cats, Beugnet F, Halos L, Scientific Editors. Ferreol: Lyon, France. 381 p. ISBN 978-2-9550805-0-4.

3. Beugnet F, Halos L, Guillot J. 2018. Textbook of clinical parasitology in dogs and cats, in Chapter Tick Infestation. Grupo Asis: Zaragoza, Spain, p. 236-249. ISBN: 978-2-9550805-2-8.

4. Beugnet F, Marié JL. 2009. Emerging arthropod-borne diseases of companion animals in Europe. Veterinary Parasitology, 163, 298-305.

5. Dantas-Torres F. 2010. Biology and ecology of the brown dog tick, Rhipicephalus sanguineus. Parasites \& Vectors, 3, 26.

6. Deplazes P, van Knapen F, Schweiger A, Overgaauw PA. 2011. Role of pet dogs and cats in the transmission of helminthic zoonoses in Europe, with a focus on echinococcosis and toxocarosis. Veterinary Parasitology, 182, 41-53.

7. Dryden MW, Hodgkins E. 2010. Vector-borne diseases in pets: the stealth health threat. Compendium on Continuing Education, 32(6), E1-E4. 
8. Estrada-Peña A, Pfäffle M, Baneth G, Kleinerman G, Petney TN. 2017. Ixodoidea of the Western Palaearctic: a review of available literature for identification of species. Ticks and TickBorne Diseases, 8, 512-525.

9. Geurden T, Becskei C, Six R, Maeder S, Latrofa M, Otranto D, Farkas R. 2018. Detection of tick-borne pathogens in ticks from dogs and cats in different European countries. Ticks and Tick Borne Diseases, 9, 1431-1436.

10. Jongejan F, Uilenberg G. 2004. The global importance of ticks. Parasitology, 129, S3-S14.

11. Hornok S, Grima A, Takács N, Kontschán J. 2018. Infestation of Rhipicephalus sanguineus sensu lato on cats in Malta ticks and tick-borne diseases, 9, 1120-11248.

12. Kunkle B, Daly S, Dumont P, Drag M, Larsen D. 2014. Assessment of the efficacy of orally administered afoxolaner against Rhipicephalus sanguineus sensu lato. Veterinary Parasitology, 201, 226-228.

13. Marchiondo AA, Holdsworth PA, Fourie LJ, Rugg D, Hellmann K, Snyder DE, Dryden MW. 2013. World Association for the Advancement of Veterinary Parasitology (W.A.A.V.P.) second edition: Guidelines for evaluating the efficacy of parasiticides for the treatment, prevention and control of flea and tick infestations on dogs and cats. Veterinary Parasitology, 194, 8497.

14. Otranto D, Brianti E, Latrofa MS, Annoscia G, Weigl S, Lia RP, Gaglio G, Napoli E, Giannetto S, Papadopoulos E, Mirò G, Dantas-Torres F, Bain O. 2012. On a Cercopithifilaria sp. transmitted by Rhipicephalus sanguineus: a neglected, but widespread filarioid of dogs. Parasites \& Vectors, 5, 1.

15. Traversa D. 2012. Pet roundworms and hookworms: a continuing need for global warming. Parasites \& Vectors, 5, 91-110.

16. Thomas JE, Staubus L, Goolsby JL, Reichard MV. 2016. Ectoparasites of free-roaming domestic cats in the central United States. Veterinary Parasitology, 228, 17-22.

17. Uspensky I. 2009. Attachment of nymphal Rhipicephalus sanguineus (Acari: Ixodidae) to a human in an urban area followed by severe adverse reaction shortly before drop-off. Folia Parasitologica, 56, 67-69.

18. Zhang J, Liu Q, Wang D, Li W, Beugnet F, Zhou J. 2017. Epidemiological survey of ticks and tick-borne pathogens in pet dogs in south-eastern China. Parasite, 24, 35.

Cite this article as: Tielemans E, Pfefferkorn A \& Viljoen A. 2021. Efficacy of a novel topical combination of esafoxolaner, eprinomectin and praziquantel against Rhipicephalus sanguineus in cats. Parasite 28, 24.

\section{PARASTTE}

An international open-access, peer-reviewed, online journal publishing high quality papers on all aspects of human and animal parasitology

Reviews, articles and short notes may be submitted. Fields include, but are not limited to: general, medical and veterinary parasitology; morphology, including ultrastructure; parasite systematics, including entomology, acarology, helminthology and protistology, and molecular analyses; molecular biology and biochemistry; immunology of parasitic diseases; host-parasite relationships; ecology and life history of parasites; epidemiology; therapeutics; new diagnostic tools.

All papers in Parasite are published in English. Manuscripts should have a broad interest and must not have been published or submitted elsewhere. No limit is imposed on the length of manuscripts.

Parasite (open-access) continues Parasite (print and online editions, 1994-2012) and Annales de Parasitologie Humaine et Comparée (1923-1993) and is the official journal of the Société Française de Parasitologie. 